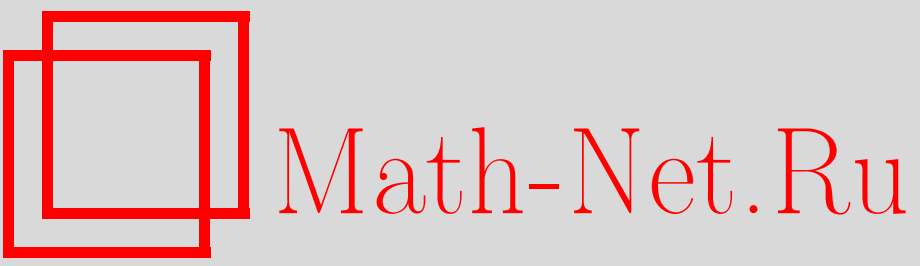

М. Д. Ковалёв, Что такое шарнирный механизм? И что же доказал Кемпе?, Итоги науки и техн. Сер. Соврем. мат. и ее прил. Темат. обз., 2020, том 179, 16-28

DOI: https://doi.org/10.36535/0233-6723-2020-179-16-28

Использование Общероссийского математического портала Math-Net.Ru подразумевает, что вы прочитали и согласны с пользовательским соглашением

http://www. mathnet.ru/rus/agreement

Параметры загрузки:

IP: 54.162 .27 .143

26 апреля 2023 г., 18:05:24 


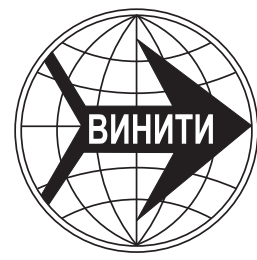

ИТОГИ НАУКИ И ТЕХНИКИ.

Современная математика и ее приложения.

Тематические обзоры.

Том 179 (2020). С. 16-28

DOI: $10.36535 / 0233-6723-2020-179-16-28$

УДК 514.8, 531.1

\title{
ЧТО ТАКОЕ ШАРНИРНЫЙ МЕХАНИЗМ? \\ И ЧТО ЖЕ ДОКАЗАЛ КЕМПЕ?
}

(c) 2020 г. $\quad$ М. Д. КОВАЛЕВ

\begin{abstract}
АннотАция. Долгое время считалось, что Альфред Кемпе доказал (1876) теорему о возможности вычерчивания по частям произвольной плоской алгебраической кривой с помощью шарнирных механизмов. Однако в конце XX века специалисты по алгебраической геометрии, передоказав и развив этот результат на современном языке, стали утверждать, что рассуждения Кемпе содержат существенные пробелы и даже ошибки. Автор считает эти обвинения необоснованными. Статья посвящена доказательству этой точки зрения. Попутно критически разбирается понимание шарнирного механизма и его конфигурационного пространства в современных математических работах.
\end{abstract}

Ключевые слова: плоский шарнирный механизм, конфигурационное пространство механизма, теорема Кемпе.

\section{WHAT IS A HINGE MECHANISM? AND WHAT DID KEMPE PROVE?}

\author{
(C) 2020 M. D. KOVALEV
}

\begin{abstract}
For a long time, it was believed that Alfred Kempe proved (1876) a theorem about the possibility of plotting an arbitrary plane algebraic curve by parts using hinge mechanisms. However, at the end of the 20th century, specialists in algebraic geometry, having rediscovered and developed this result in modern language, began to assert that Kempe's reasoning contained significant gaps and errors. In the author's opinion, these charges are unfounded. In this paper, the author tries to substantiate this point of view. Moreover, the notions of a hinge mechanism and its configuration space are analyzed.
\end{abstract}

Keywords and phrases: flat hinge mechanism, mechanism configuration space, Kempe theorem.

AMS Subject Classification: 52C99, 70B15, 14P99

1. Введение. Альфредом Кемпе в статье [19] был положительно решен вопрос о черчении по частям произвольной плоской алгебраической кривой с помощью шарнирных механизмов. В этой работе, носящей название «Об общем методе черчения шарнирным механизмом плоских кривых $n$-й степени», не содержится формулировок каких-либо теорем. Целый век считалось, что Кемпе доказал теорему, закрывшую тему черчения алгебраических кривых на плоскости шарнирными механизмами. Однако в середине 1970-х гг. известный американский математик У. Терстон обратил внимание на проблематику теоремы Кемпе. В устных докладах он эффектно формулировал результат: можно построить шарнирный механизм, воспроизводящий вашу подпись. Хотя Терстон не публиковал статей по механизмам, но его выступление вдохновило других. Начиная с 1998 г. появились работа М. Каповича и Дж. Миллсона [18], а также ряд статей Г. Кинга [20-22], посвященных истолкованию результата Кемпе на языке современной алгебраической геометрии. 
Их авторы утверждали, что рассуждения Кемпе содержат существенные ошибки, и это мнение получило распространение среди математиков (см. $[9,10,14])$.

Однако при внимательном рассмотрении претензий к Кемпе выясняется, что они носят надуманный характер. Дело в том, что результат Кемпе имеет локальный характер. Авторы же вышеназванных работ получили результаты относительно конфигурационного пространства шарнирных механизмов в целом. Пытаясь уличить Кемпе в неточности, они приписывают Кемпе свою формулировку его основного результата и указывают на недостаточность его аргументов для доказательства их теоремы.

Формализуя результат Кемпе, Каповичу и Миллсону, Кингу, а вслед за ними и другим авторам (см. $[9,10,14,17])$ пришлось ввести математическое понятие шарнирного механизма и его конфигурационного пространства. Однако введенные ими понятия продиктованы лишь удобством применения математического инструментария и не согласуются с пониманием этих терминов в механике и теории механизмов. Так, конфигурационное пространство механизма у этих авторов может быть несвязным и содержать изолированные точки в качестве компонент. Я уже давно продвигаю (см. [4-7,23]) математическую формализацию геометрии шарнирных конструкций, согласованную идейно и терминологически с теорией механизмов и строительной механикой. В разделе 2 статьи будет приведена эта формализация, кстати, позволяющая естественнее сформулировать и результаты работ $[18,20-22]$. Также будут критически проанализированы определения работ М. Каповича, Дж. Миллсона и Г. Кинга. В разделе 3 воспроизводятся рассуждения статьи [19] Кемпе. В разделе 4 приведены результаты современных работ в направлении теоремы Кемпе. В разделе 5 подробно обсуждаются претензии, выдвинутые к Кемпе. Сформулирована теорема, к которой с небольшими уточнениями приводят построения Кемпе.

2. Формализация понятия шарнирного механизма и его конфигурационного пространства. В инженерии первично деление конструкций на неподвижные, называемые фермами, и подвижные, называемые механизмами. Давид Гильберт в своих лекциях по наглядной геометрии [2] так определял механизм: «Плоским шарнирным механизмом называется всякая плоская система жестких стержней, частично соединенных между собой или скрепленных с неподвижными точками плоскости, вокруг которых они могут вращаться, так что вся система еще сохраняет подвижность в ее плоскости». Это общепринятое (см. $[12,16])$ определение является не математическим, а описательным. Его, конечно, достаточно для ответа на вопрос: является ли данная конструкция из жестких стержней плоским шарнирным механизмом? Однако оно не дает способа описания индивидуального механизма и различения механизмов между собой. Эта неполнота должна быть преодолена в точном определении.

Естественно начать с определения строения механизма. Мы считаем наши механизмы составленными из стержней, несущих на своих концах шарниры. Структуру такого механизма задаем графом $G(V, E)$ без петель и кратных ребер, вершины которого отвечают шарнирам, а ребра рычагам (стержням $)^{1}$. Граф $G(V, E)$ обладает вершинами двух видов: крестиками мы обозначаем вершины, отвечающие шарнирам, неподвижным в механизме (закрепленным), кружочками подвижным в механизме (свободным) шарнирам.

На граф $G(V, E)$ шарнирного механизма естественно наложить следующие условия:

(a) граф $G(V, E)$ связен,

(b) вершины-крестики смежны лишь вершинам-кружочкам,

(c) подграф графа $G(V, E)$ на вершинах-кружочках связен,

(d) условие, вытекающее из подвижности свободных шарниров; из него следует, например, что каждый кружочек смежен не более чем одному крестику.

Граф $G(V, E)$, для которого выполнены все эти условия, мы называем шарнирной структурной схемой (ШСС) механизма. Условия (а), (c) выделяют индивидуальный механизм, без них мы получаем несколько механизмов, движущихся независимо один от другого. Условие (b) вводится из-за того, что нет нужды задавать расстояния между закрепленными шарнирами, что вполне

\footnotetext{
${ }^{1}$ Заметим, что в теории механизмов структурной схемой обычно считают граф, вершины которого отвечают звеньям шарнирного механизма, а ребра - вращательным кинематическим парам.
} 
прояснится после введения понятия закрепленной шарнирной схемы. Условие (d) введено, чтобы исключить неподвижные шарниры из числа свободных; если нас интересуют не только механизмы, но и фермы такой структуры, то вводить его не нужно. В этой общей ситуации ШСС, удовлетворяющую условию (d), будем называть приведенной структурной схемой механизма.

Абстрактные математики (см. $[18,20-22])$ также начинают с графа, подобного $G(V, E)$, но не накладывают на него никаких условий, кроме разве лишь конечности и отсутствия петель. У них он может быть и несвязным. Мы же с самого начала стараемся наложить условия, диктуемые задачей изучения шарнирных механизмов и отсекающие пустопорожнее теоретизирование вроде формулирования утверждения о том, что если граф $G(V, E)$ несвязен, то пространство его реализаций представляет собой прямое произведение пространств реализаций его связных компонент или учета в теоремах случая, в котором множество положений одного шарнира «шарнирного механизма» может совпадать со всей плоскостью, что, очевидно, получается при наличии компоненты связности графа $G(V, E)$, не содержащей закрепленных шарниров. Я уверен, что это один из тех случаев, когда «полная общность», которой стараются придерживаться авторы, кроме них самих больше никому не нужна.

Закрепленной шарнирной схемой (ЗШС) называем ШСС, для которой заданы положения закрепленных шарниров в плоскости. Положения закрепленных шарниров мы считаем попарно несовпадающими. Пусть ЗШС имеет $n$ закрепленных и $m$ свободных шарниров и $r$ рычагов $(n \geqslant 1, m \geqslant 1, r \geqslant 1)$. Тогда ЗШС отвечают два евклидовых пространства параметров: $\mathbb{R}^{2 m}$ пространство положений свободных шарниров в плоскости и $\mathcal{R}^{r}$ - пространство квадратов длин рычагов. Пусть $p_{i}$ - радиус-вектор $i$-го шарнира в плоскости, $d_{i j}$ - квадрат длины рычага, соединяющего смежные $i$-й и $j$-й шарниры в графе $G(V, E)$. Ключевым для геометрии шарнирных конструкций является так называемое рычажное отображение

$$
F: \mathbb{R}^{2 m} \rightarrow \mathcal{R}^{r}, \quad d_{i j}=\left(p_{i}-p_{j}\right)^{2}, \quad\{i j\} \in E .
$$

Это отображение ставит в соответствие положениям свободных шарниров квадраты длин рычагов и называется рычажным (в англоязычной литературе - «rigidity mapping» или «edge function»; см. $[11,13,15])$. Точку $\boldsymbol{d}=\left\{d_{i j}\right\} \in \mathcal{R}^{r}$ мы называем, придавая вполне определенный смысл термину и понятию кинематической схемы ${ }^{1}$ из теории механизмов, кинематической шарнирной схемой (КШС), а точку $\boldsymbol{p}=\left\{p_{i}\right\} \in \mathbb{R}^{2 m}$ - шарнирником. С инженерной точки зрения, шарнирник есть либо определенное положение шарнирного механизма, либо шарнирная ферма. Неодноточечная компонента $K \subset \mathbb{R}^{2 m}$ связности полного прообраза $F^{-1}(\boldsymbol{d}) \mathrm{KШC} \boldsymbol{d}$ представляет собой множество всех положений, или конфигурационное пространство шарнирного механизма. Таким образом, мы отождествляем шарнирный механизм с его связным конфигурационным пространством.

Полный прообраз $F^{-1}(\boldsymbol{d})$ точки при рычажном отображении называем конфигурационным пространством КШС $\boldsymbol{d}$. При таком подходе каждой компоненте связности полного прообраза $F^{-1}(\boldsymbol{d})$ отвечает определенное шарнирное устройство. Если компонента связности одноточечна, то это устройство представляет собой шарнирную ферму. Шарнирная ферма не допускает непрерывного движения свободных шарниров без изменения длин рычагов и положений закрепленных шарниров.

Естественно, модель несет существенные черты идеализации. При движении нашего шарнирного механизма различные его рычаги могут пересекаться, а несмежные шарниры совмещаться. Подчеркнем еще раз, что наше определение шарнирного механизма согласуется с его пониманием специалистами по теории механизмов. Вот какое определение механизма читаем у Артоболевского (см. [1]): «Механическая система тел, предназначенная для преобразования движений одного или нескольких тел в требуемые движения других тел, называется механизмом». Неотъемлемыми свойствами механизма считаются его подвижность и возможность непрерывно перевести его из произвольного положения в любое другое его положение. Геометрически это означает положительность размерности и связность его конфигурационного пространства.

\footnotetext{
${ }^{1}$ В сборнике терминов [8] по теории механизмов дается такое определение: «Кинематическая схема механизма: структурная схема механизма с указанием размеров звеньев, необходимых для кинематического анализа механизма».
} 
a)

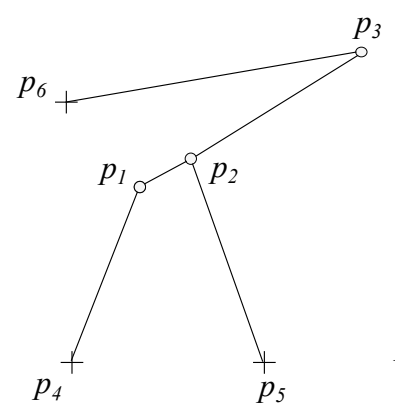

b)

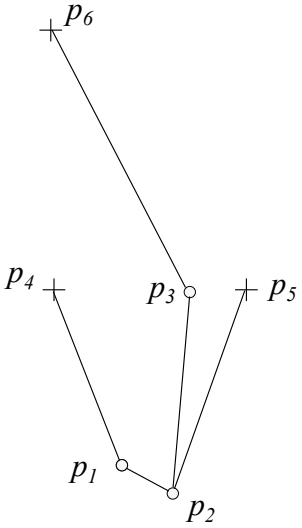

Рис. 1

Авторы же работ $[18,20]$ и вслед за ними другие математики называют конфигурационным пространством шарнирного механизма то, что я называю конфигурационным пространством его КШС, т.е. множество $F^{-1}(\boldsymbol{d})$. С точки зрения математики это естественнее и удобнее, поскольку это множество - в отличие от его связной компоненты - всегда является алгебраическим множеством. Но такая терминология и такое пренебрежение смыслом слов вполне способно запутать механиков. Приведем пример. На рисунке 1 изображены два шарнирных механизма (a) и (b) с одной и той же кинематической схемой. Механизмы состоят из четырехзвенника $p_{4} p_{1} p_{2} p_{5}$, к которому присоединена двухповодковая группа $p_{6} p_{3} p_{2}$. Если рычаг $p_{1} p_{2}$ достаточно короток, то четырехзвенники, отличающиеся отражением относительно прямой $p_{4} p_{5}$, нельзя непрерывно перевести один в другой. В терминологии машиноведов речь идет о двух сборках шарнирного механизма. Однако, уменьшая длины рычагов $p_{2} p_{3}$ и $p_{3} p_{6}$, можно добиться, чтобы сборка (b) стала фермой. Это наступит, когда рычаги $p_{2} p_{3}$ и $p_{3} p_{6}$ окажутся на одной прямой, и круг с центром $p_{6}$ радиуса равного сумме длин этих рычагов будет иметь лишь одну общую точку с траекторией шарнира $p_{2}$ четырехзвенника, чего можно добиться выбором положения закрепленного шарнира $p_{6}$. Сборка же (а) при этом останется механизмом.

Разумно ли механизм и ферму называть разными сборками одного шарнирного механизма, как это делают машиноведы? Или называть эту пару конструкций, непрерывно не переводимых одна в другую, шарнирным механизмом, что вытекает из определений абстрактных математиков? При нашем подходе здесь речь идет о двух шарнирных устройствах - механизме и ферме с одной и той же кинематической схемой.

Отметим еще разноголосицу в определениях абстрактных математиков. Миллсон и Капович [18] начинают с графа $G^{\prime}(V, E)$ с вершинами одного сорта, не накладывая на него никаких условий кроме отсутствия петель. Далее они выделяют в $G^{\prime}(V, E)$ множество $W$ вершин, отвечающих закрепленным шарнирам. При этом закрепленные шарниры могут оказаться соединенными рычагом. Абстрактным отмеченным механизмом $\mathcal{L}$ они называют тройку $\left(G^{\prime}(V, E), l, W\right)$, где $l-$ набор положительных длин, поставленных в соответствие ребрам графа $G^{\prime}(V, E)$. Фиксируя закрепленные шарниры, они получают рычажное отображение $F$, и $F^{-1}(\boldsymbol{d})$ называют относительным конфигурационным пространством (relative configuration space) механизма $\mathcal{L}$ (здесь $\boldsymbol{d}$ отвечает набору $l$ ). В случае отсутствия закрепления $W$ они называют множество реализаций графа с заданными длинами ребер в плоскости конфигурационным пространством механизма $\mathcal{L}$. Есть у них еще и базированные механизмы (based linkages), у которых закреплены лишь две вершины одного ребра. Соответствующее конфигурационное пространство они называют пространством 


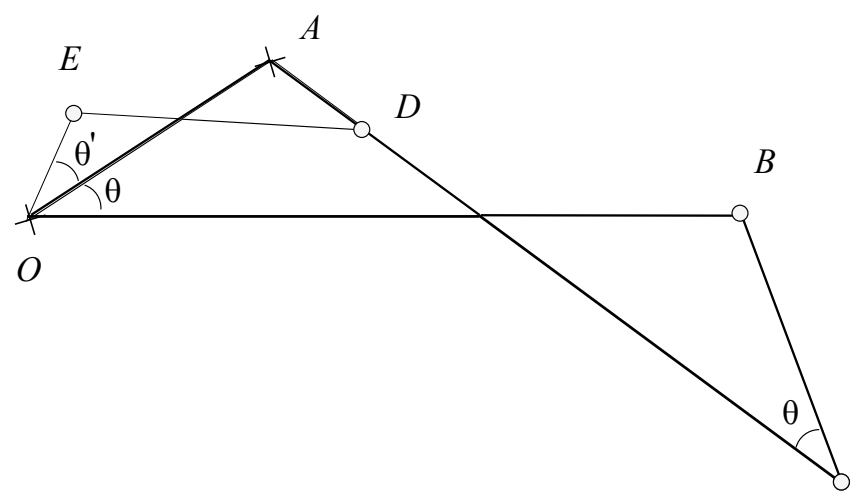

Рис. 2

C

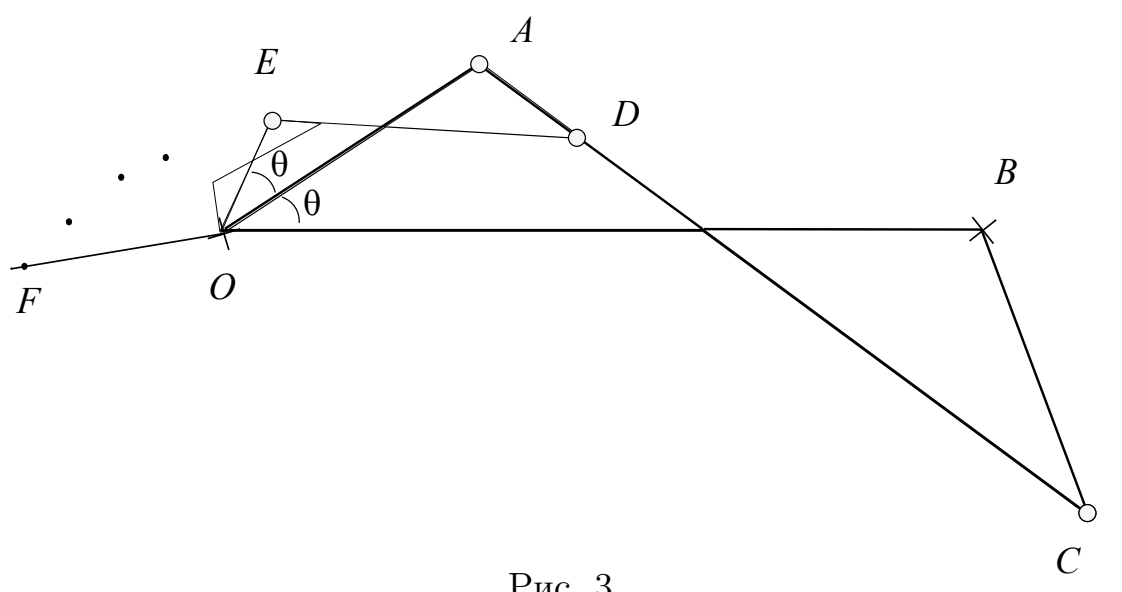

Рис. 3

модулей (moduli space) шарнирного механизма. Конфигурационное пространство последних всегда имеет симметрию, порожденную отражением плоскости от прямой, содержащей два закрепленных шарнира. Кинг [20] на граф $G(V, E)$ накладывает лишь условие конечности. Он называет механизмами конструкции, шарниры которых связаны не только рычагами, но и веревками. Обычные же механизмы он называет классическими. Необходимость введения таких обобщенных механизмов сомнительна, ибо веревку можно заменить двумя рычагами длины, равной половине длины веревки, и это не повлияет на возможности рисовать кривые шарнирами механизма. Авторы работы [17] помимо конечности и отсутствия петель требуют связности графа $G(V, E)$, но не требуют связности его подграфа на вершинах, отвечающих свободным шарнирам. Механизмом они называют совокупность графа $G(V, E)$ и набора длин его ребер, для которой имеются реализации этого метрического графа в плоскости.

3. Рассуждения Кемпе. Для понимания дальнейшего необходимо воспроизвести рассуждения Кемпе. Хотя Кемпе, как было сказано, и не формулировал теоремы, мы приведем его результат в виде «наивной» теоремы, как его понимали на протяжении столетия.

Теорема 1. Всякий достаточно малый кусок произволъной плоской алгебраической кривой представляет собой множество положений шарнира плоского шарнирного механизма.

Доказательство Кемпе заключается в указании способа построения нужного механизма из простейших механизмов-кирпичиков, выполняющих определенные алгебраические действия. Сначала перечислим эти механизмы-кирпичики. 


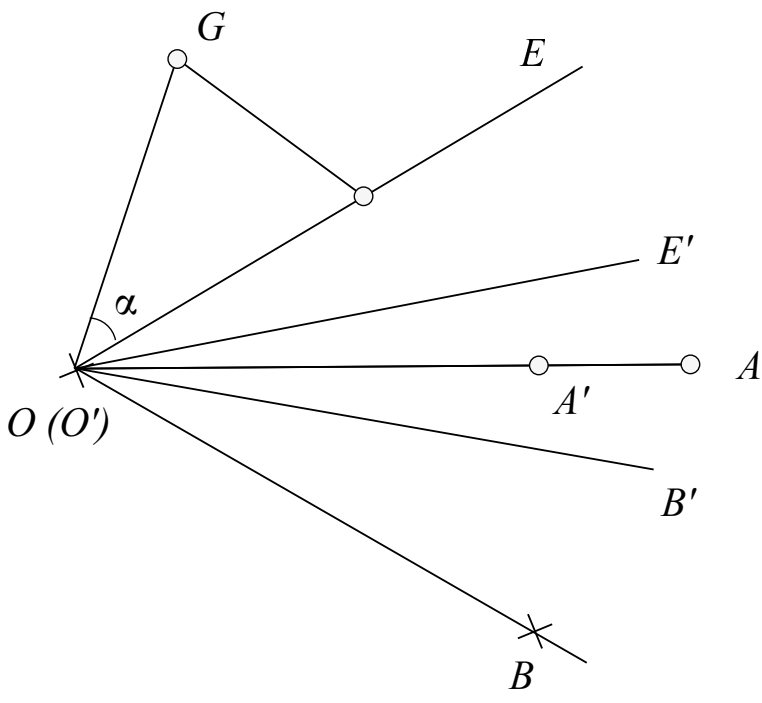

Рис. 4

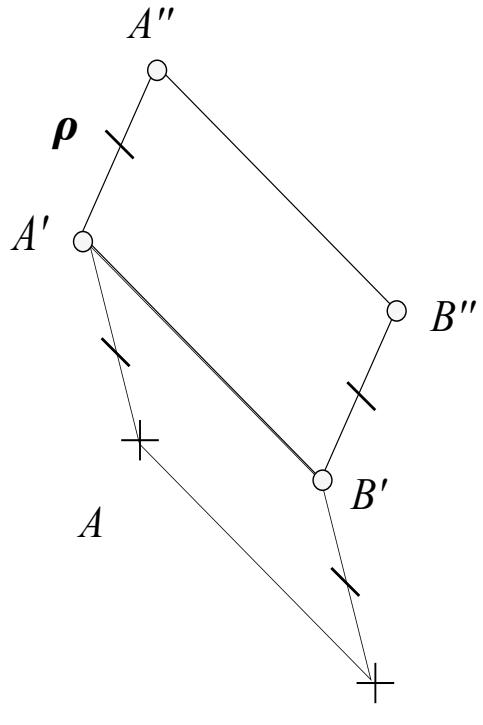

$B$

Рис. 5

Механизм сдвоенного антипараллелограмма - опрокидыватель (см. рис. 2). Механизм состоит из четырехзвенника $O A C B$, в котором закреплены шарниры $O$ и $A$, и равны длины противоположных рычагов, два из которых $(O B$ и $A C)$ пересекаются. Такой механизм называют антипараллелограммом, при его движении угол $\theta$ меняется. $\mathrm{K}$ этому антипараллелограмму приделан меньший подобный ему антипараллелограмм $O A D E$. Он откладывает угол $\theta^{\prime}=-\theta$ по другую сторону от прямой $O A$.

Механизм умножитель получается, если в первом антипараллелограмме предыдущего механизма закрепить шарниры $O$ и $B$, а затем пристроить к нему нужное число антипараллелограммов, как показано на рис. 3. С его помощью можно получить угол $\angle B O F=n \angle B O A$ для произвольного натурального $n$.

Механизм накопитель. Чтобы получить сумму углов, нужно от луча $O B$ (рис. 4) отложить складываемые углы $\angle B O B^{\prime}$ и $\angle B O E^{\prime}$ и соединить их штрихованным опрокидывателем, взяв его шарнир $B^{\prime}$ на луче $O B^{\prime}$, а шарнир $E^{\prime}$ - на луче $O E^{\prime}$. При этом рычаг $O A^{\prime}$ окажется на биссектрисе угла $B^{\prime} O E^{\prime}$, т.е. $\angle B O A^{\prime}=\frac{1}{2}\left(\angle B O B^{\prime}+\angle B O E^{\prime}\right)$. Остается удвоить этот угол с помощью нештрихованного опрокидывателя - умножителя, присоединив его шарниром $A$ к лучу $O A^{\prime}$ и шарниром $B$ к лучу $O B$. Рычаг $O E$ добавленного нештрихованного опрокидывателя укажет сторону угла $\angle B O E=\angle B O B^{\prime}+\angle B O E^{\prime}$.

Мы также можем прибавить постоянный угол $|\alpha|<\pi / 2$, как показано на рис. 4 , присоединяя к конечному рычагу $O E$ двумя рычагами шарнир $G$. Если нужно, повторяя это построение, можем добавить угол произвольной величины.

Последний наш механизм - переносчик, состоящий из двух параллелограммов с боковыми сторонами длины $\rho$, как показано на рис. 5. Он позволяет параллельно переносить рычаг $A B$ в рычаг $A^{\prime \prime} B^{\prime \prime}$, причем точка $A^{\prime \prime}$ может быть помещена в любую точку плоскости, отстоящую от $A$ не более чем на $2 \rho$.

Опишем теперь построение механизма Кемпе. Пусть $p$-точка на алгебраической кривой. Сдвинув, если нужно, декартову прямоугольную систему координат $O X Y$, можно считать, что точка $p$ не совпадает с ее началом, и можно построить шарнирный ромб (см. рис. 6) с рычагами $|O A|=|O B|=a$ постоянной длины. Опираясь на его рычаги $O A$ и $O B$, построим наш шарнирный механизм. Пусть $\angle X O A=\theta, \angle X O B=\varphi$, и эти углы меняются при движении точки $p$ по 


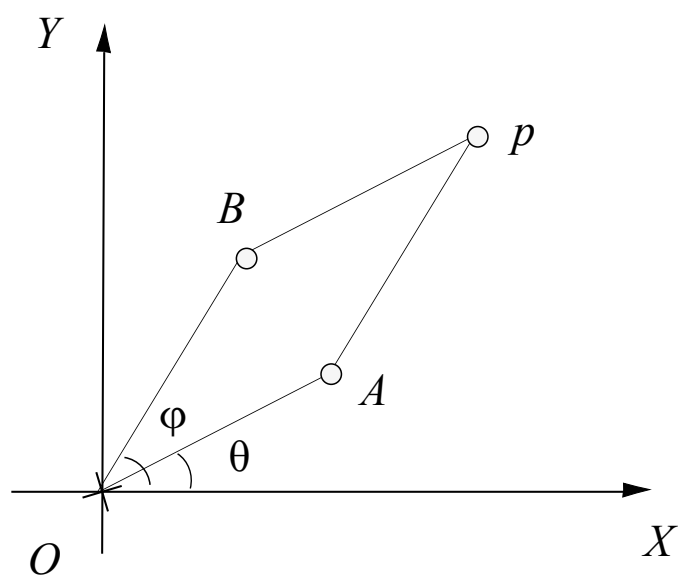

Рис. 6

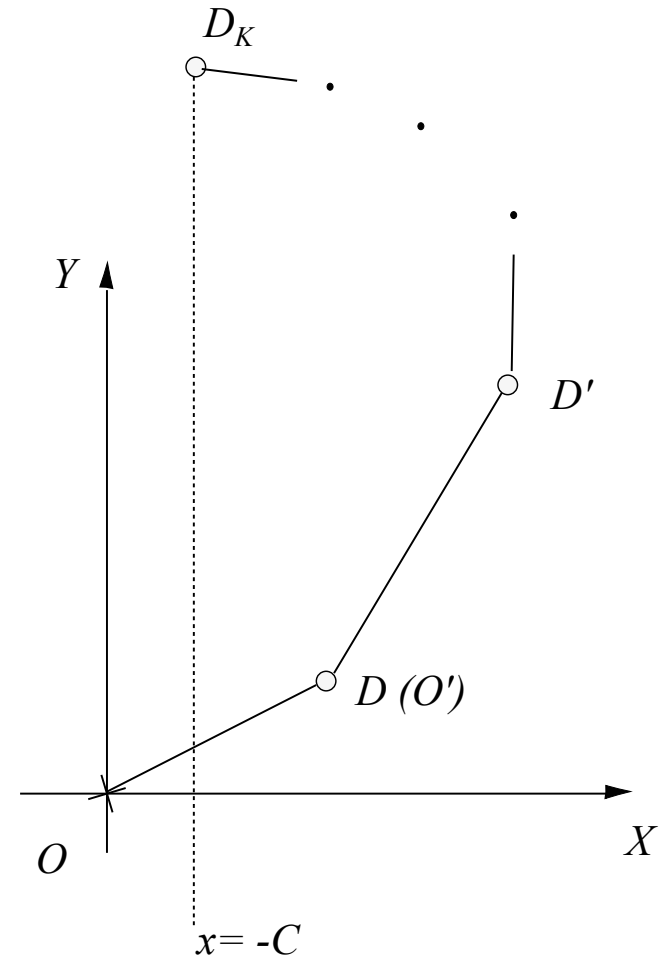

Рис. 7

алгебраической кривой, заданной в нашей системе координат уравнением

$$
f(x, y)=\sum_{k l} A_{k l} x^{k} y^{l}=0,
$$

где $k, l$ - неотрицательные целые. Выпишем координаты точки $p$ :

$$
\begin{aligned}
& x=a(\cos \theta+\cos \varphi), \\
& y=a\left(\cos \left(\theta-\frac{\pi}{2}\right)+a \cos \left(\varphi-\frac{\pi}{2}\right) .\right.
\end{aligned}
$$

Подставим эти выражения в уравнение кривой и далее, применяя формулу $\cos \alpha \cos \beta=\frac{1}{2}[\cos (\alpha-$ $\beta)+\cos (\alpha+\beta)]$, придем к уравнению вида

$$
f(x, y)=\sum_{r, s} B_{r s} \cos \left(r \varphi+s \theta+\chi_{r s}\right)+C=0,
$$

где $r, s$ - целые, не равные нулю одновременно, $C$-постоянная, зависящая от $A_{k l}$ и $a$, а $\chi_{r s}-$ постоянный угол. За счет выбора этого угла можно считать коэффициенты $B_{r s}$, выражающиеся через $A_{k l}, a$, положительными.

С помощью механизмов накопителя и умножителя для каждого члена последней суммы можно построить такой рычаг $O D_{r s},\left|O D_{r s}\right|=B_{r s}$, что $\angle X O D_{r s}=r \varphi+s \theta+\chi_{r s}$. Составим теперь с помощью переносчика из рычагов $O^{\prime} D_{r s}^{\prime}$ (представляющих собой параллельно перенесенные рычаги $O D_{r s}$ ) цепь с конечным шарниром $D_{K}$, как показано на рис. 7 . Абсцисса шарнира $D_{K}$ равна

$$
\sum_{r, s} B_{r s} \cos \left(r \varphi+s \theta+\chi_{r s}\right)=f(x, y)-C .
$$

Точка $p$ лежит на нашей кривой тогда и только тогда, когда $f(x, y)=0$, а следовательно, тогда и только тогда, когда конечный шарнир $D_{K}$ цепи лежит на вертикальной прямой $x=-C$. Остается посадить шарнир $D_{K}$ на шарнир инверсора Поселье (см. $\left.[2,9]\right)$, совершающий прямолинейное 
движение по этой прямой, и мы получим механизм, шарнир которого, совпадающий с вершиной $p$ ромба, движется по нашей алгебраической кривой.

4. Результаты современных работ. Алгебраическим множеством называем множество общих нулей совокупности многочленов относительно переменных - декартовых координат точки в $\mathbb{R}^{k}$. Аналитический изоморфизм подмножеств евклидовых пространств - гомеоморфизм, осуществляемый сужениями аналитических (т.е. представимых покоординатно локально сходящимися степенными рядами) отображений.

Теорема 2 (М. Капович, Дж. Миллсон). Для любого компактного алгебраического множества $X \subset \mathbb{R}^{k}$ найдется КШС, конфигурационное пространство которой аналитически изоморфно конечному набору непересекающихся копий множества $X$.

Прямым следствием из этой теоремы является следующее утверждение.

Теорема 3. Для любого связного гладкого компактного многообразия X найдется шарнирный механизм, конфигурационное пространство которого гладко гомеоморфно (дифбеоморфно) многообразию $X$.

В отличие от результата Кемпе эти теоремы имеют характер результатов «в целом». Их доказательство опирается на видоизмененный метод Кемпе построения нужного механизма из простейших.

Имеется и следующий результат. Рассмотрим кусок $K$ полиномиально параметризованной кривой $f:[a, b] \rightarrow \mathbb{R}^{2}$ (отметим, что полиномиально параметризованная кривая является алгебраической).

Теорема 4 (М. Капович, Дж. Миллсон). Кривая $K$ может быть прочерчена шарниром подходящим образом выбранного плоского шарнирного механизма, а именно, закон двиюения этого шарнира есть $f(t), a \leqslant t \leqslant b$.

Поскольку любую непрерывную на отрезке $[a, b]$ функцию можно сколь угодно точно приближать многочленом, то утверждение Терстона о подписи, рисуемой без отрыва ручки от листа бумаги, сводится к этой теореме.

Г. Кинг распространил эти результаты на случай механизмов в $\mathbb{R}^{d}$ при $d>2$. Кинг также доказал, что множество положений шарнира для плоской КШС может быть любым компактным полуалгебраическим множеством плоскости. Полуалгебраическое множество-это конечное объединение множеств решений конечных систем полиномиальных уравнений и полиномиальных неравенств. Всякое алгебраическое множество является и полуалгебраическим. Обратное неверно. Например, круг, будучи полуалгебраическим, не является алгебраическим множеством. Заметим, что, как следует из теоремы Тарского-Зайденберга (см. [3]), проекция полуалгебраического множества на координатное подпространство является полуалгебраическим множеством. Проекция алгебраического множества далеко не всегда есть алгебраическое множество.

Несмотря на общность теорем, доказанных Кингом, остается открытым следующий естественный вопрос (см. [14]): является ли произвольное связное компактное полуалгебраическое множество плоскости множеством положений некоторого шарнира плоского шарнирного механизма?

Действительно, хотя Кингом доказано, что любое связное компактное полуалгебраическое множество является множеством положений некоторого шарнира для заданной КШС. Однако оно может являться проекцией несвязного конфигурационного пространства КШС и быть объединением положений нашего шарнира для нескольких шарнирных механизмов.

5. Разбор претензий к Кемпе. Попытаемся формализовать результат Кемпе. Пусть $K-$ конфигурационное пространство КШС $\boldsymbol{d}$, а $K_{i}$ - конфигурационное пространство плоского шарнирного механизма, отвечающего этой КШС, т.е. неодноточечная компонента связности $K$. Далее, пусть $K_{i}^{j}$ - множество положений $j$-го шарнира механизма $K_{i}$. Иными словами, $K_{i}^{j}-$ проекция конфигурационного пространства $K_{i}$ механизма на плоскость $\mathbb{R}_{j}^{2}$ положений $j$-го шарнира.

Теорема, которую проще всего было бы сформулировать на основе работы Кемпе, имеет следующий вид. 


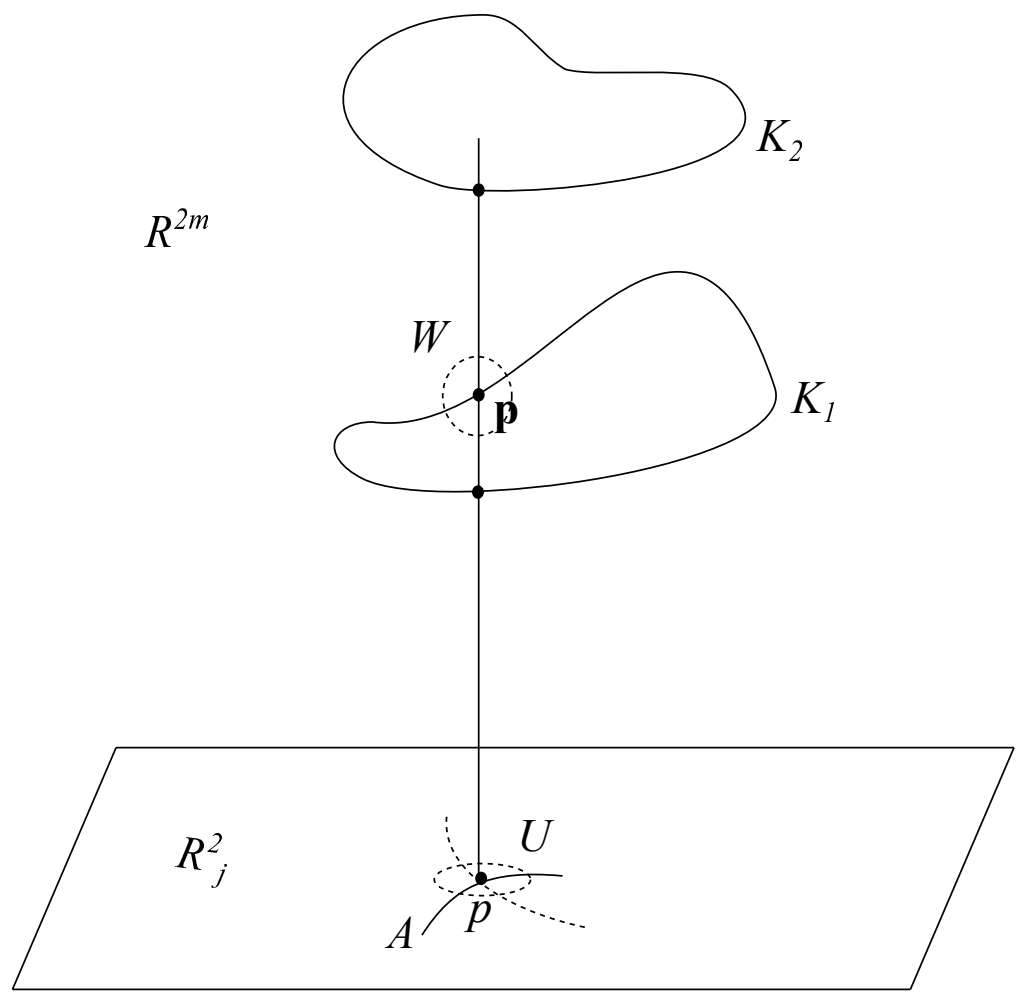

Рис. 8. Положению $j$-го шарнира $p$ отвечают два положения шарнирного механизма $K_{1}$ и одно положение механизма $K_{2}$.

Теорема 5. Для произвольной плоской алгебраической кривой $A$ и точки $p \in A$ найдутся окрестность $U$ точки р и механизм с шарниром $p_{j}$, для которого $K_{i}^{j} \cap U=A \cap U$.

Главное затруднение заключается в том, что в точку $p$ могут проектироваться различные точки конфигурационного пространства механизма $K_{i}$, а также точки конфигурационных пространств других механизмов с этой же кинематической схемой (см. рис. 8). Естественно, может оказаться, что $K_{i}^{j} \cap U \supset A \cap U$, и тем более $K^{j} \cap U \supset A \cap U$, где $K^{j}$ - проекция $K$ на плоскость положений $j$-го шарнира. Тогда теорема 5 окажется неверной.

С последней возможностью вынуждены бороться те, кто принимает точку зрения на конфигурационное пространство шарнирного механизма, насаждаемую алгебраическими геометрами. Такое понимание конфигурационного пространства механизма игнорирует его кинематическую природу. Если мы чертим кривую $A$ шарниром $p_{j}$ механизма $K_{i}$, нас совершенно не волнует, попадет ли шарнир $p_{j}$ другого механизма с той же кинематической схемой в начальную точку $p$ кривой $A$. Ведь мы же не сможем перейти к другому механизму, непрерывно двигая механизм $K_{i}$ !

Возможность $K_{i}^{j} \cap U \supset A \cap U$, однако, остается, и из рассуждений Кемпе вытекает лишь следующая теорема.

Теорема 6. Для произвольной плоской алгебраической кривой $A$ и точки $p \in A$ найдутся окрестность $U$ точки р и механизм с шарниром $p_{j}$, для которого $K_{i}^{j} \cap U \supset A \cap U$.

Надо сказать, что если $K_{i}^{j} \cap U$ одномерно, т.е. состоит из фрагментов гладких кривых, то метод рисования алгебраической кривой Кемпе работает. Достаточно лишь выбрать нужный фрагмент.

Но наиболее естественной теоремой, учитывающей кинематическую природу задачи, является следующая. Пусть механизм имеет $m$ свободных шарниров, а значит, $K \subset \mathbb{R}^{2 m}$, и пусть $\boldsymbol{p} \in$ $F^{-1}(\boldsymbol{d})$. 


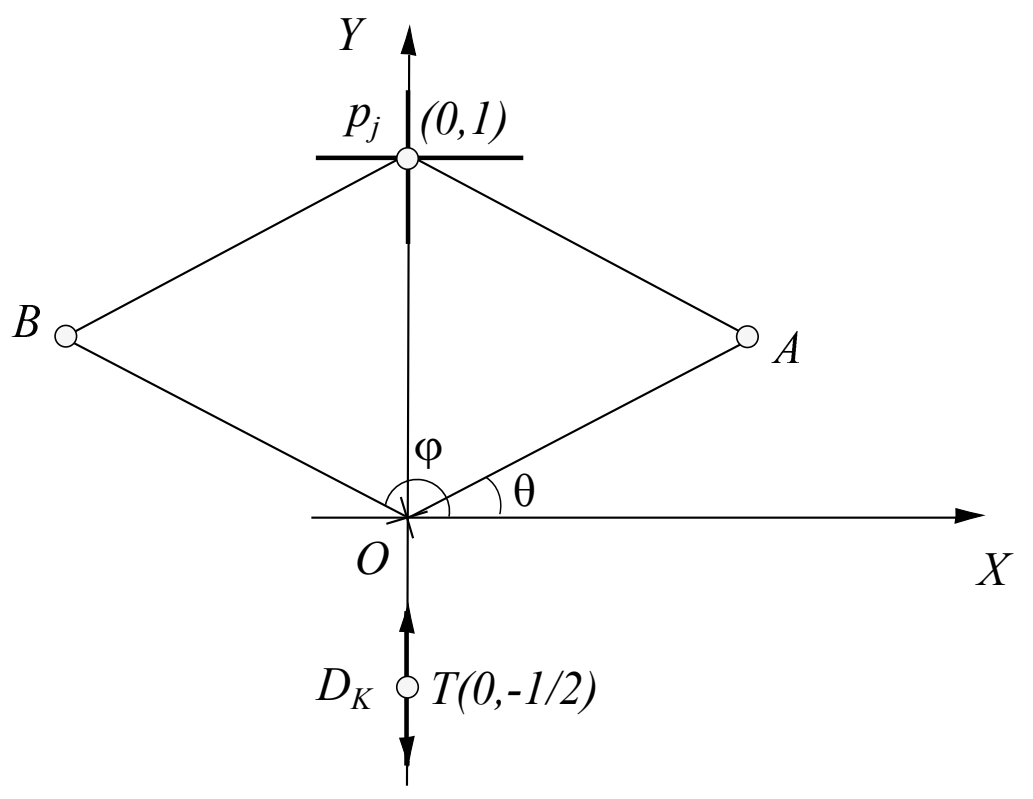

Рис. 9

Теорема 7. Для произвольной плоской алгебраической кривой $A$ и точки $p \in A$ найдется механизм с шарниром $p_{j}$ и такал окрестность $U \subset \mathbb{R}^{2}$ точки $p$, а также окрестность $W \subset \mathbb{R}^{2 m}$ точки $\boldsymbol{p}$, что $\pi\left(K_{i} \cap W\right)=A \cap U$, где $\pi$ - проекиия на плоскость положений шарнира $p_{j}$.

Из этой теоремы следует, что в случае связности $A \cap U$ каждая точка из $A \cap U$ достигается шарниром $p_{j}$ при непрерывном и небольшом движении механизма, а именно движении, не выводящем его из окрестности $W$.

Разберем подробнее претензии, выдвигаемые к работе Кемпе. Первая из них (см. [18]) относится к справедливости его в особых точках алгебраической кривой. В этих точках кривая может ветвиться, что, например, не имеет места для движения чертящего шарнира в теореме 4. На мой взгляд, эта претензия необоснованна. Действительно, в точке $p$ ветвления алгебраической кривой, которую мы чертим методом Кемпе, наблюдается интересное кинематическое явление. Движение от чертящего шарнира $p_{j}$ механизма к его шарниру $D_{K}$ (см. доказательство теоремы 1$)$, принадлежащему инверсору Поселье и перемещающемуся по отрезку прямой, передается с однозначной определенностью. В то время как движение в обратном направлении от $D_{K}$ к $p_{j}$ передается неоднозначно, с точностью до выбора ветви кривой в точке $p \in R^{2}$. Но это не означает, что в точке ветвления $p$ рассуждения Кемпе не справедливы.

Приведем соответствующие примеры. Возьмем шарнирный ромб со стороной единичной длины и вершиной в начале координат (см. рис. 9). Будем строить методом Кемпе на этом ромбе механизм, шарнир $p_{j}$ которого, совпадающий с вершиной ромба, чертит кривую $x(y-1)=0$. Пусть $\angle X O A=\theta, \angle X O B=\varphi$.

Подставляя выражения координат шарнира $p_{j}$ через углы в уравнение $x(y-1)=0$, придем к уравнению

$$
\frac{1}{2} \cos \left(2 \varphi-\frac{\pi}{2}\right)+\frac{1}{2} \cos \left(2 \theta-\frac{\pi}{2}\right)+\cos \left(\varphi+\theta-\frac{\pi}{2}\right)+\cos (\varphi+\pi)+\cos (\theta+\pi)=0 .
$$

Когда шарнир $p_{j}$ отвечающего этому уравнению механизма Кемпе, находится в точке $(0,1)$, шарнир $D_{K}$ лежит в точке $T=(0,-1 / 2)$. Если сдвигать шарнир $p_{j}$ по оси $O Y$, то шарнир $D_{K}$ будет перемещаться вверх от точки $T$. На рис. 10(а) сплошной линией показано изменение ординаты шарнира $D_{K}$ в зависимости от смещения шарнира $p_{j}$. Если сдвигать шарнир $p_{j}$ по прямой $y=1$, то шарнир $D_{K}$ будет смещаться вниз от точки $T$. Зависимость смещения $D_{K}$ от такого смещения шарнира $p_{j}$ показана сплошной линией на рис. 10(b). Пунктиром на обоих рисунках показано постоянное нулевое значение абсциссы шарнира $D_{K}$. Итак, при ненулевой скорости движения 


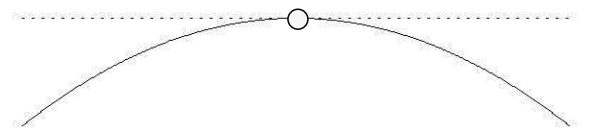

a)

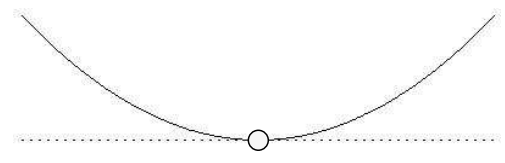

b)

Рис. 10. Кружочком обозначено положение шарнира $D_{K}$, в котором он совпадает с точкой $T$.

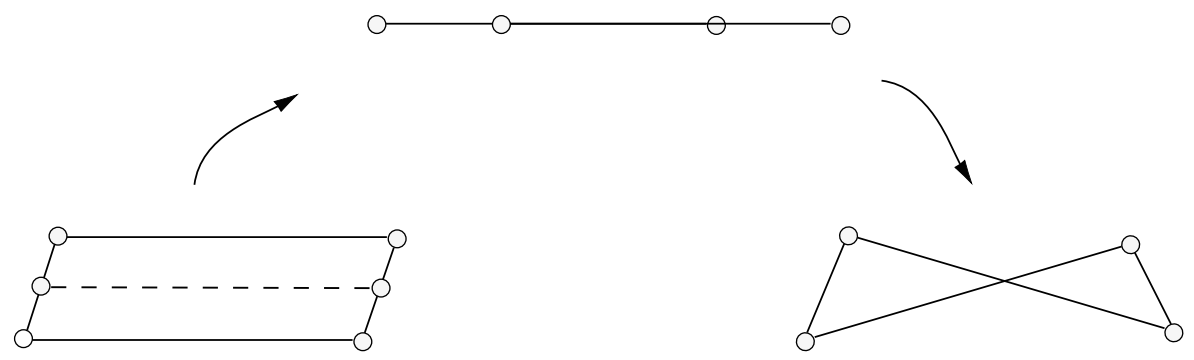

Рис. 11. Рычаг, обозначенный пунктиром, предотвращает переход параллелограмма в антипараллелограмм.

шарнира $p_{j}$, в точке $T$ скорость шарнира $D_{K}$ равна нулю. Движение механизма из положения $\boldsymbol{p}$, в котором шарнир $D_{K}$ лежит в точке $T$, существенно неоднозначно. Если шарнир $D_{K}$ двигать из положения $\boldsymbol{p}$ вверх, то шарнир $p_{j}$ движется по вертикали. Если шарнир $D_{K}$ двигать из положения $\boldsymbol{p}$ вниз, то шарнир $p_{j}$ движется по горизонтали. Однако, например, при движении $D_{K}$ из положения $\boldsymbol{p}$ вниз, шарнир $p_{j}$ может начать двигаться как влево, так и вправо. Если механизм находился в покое в положении $\boldsymbol{p}$, то совершенно неясно - в какую сторону он начнет двигаться? Заметим еще, что при приближении шарнира $D_{K}$ к точке $T$ двигать этот шарнир будет все труднее, ибо постоянной ненулевой скорости его движения должна отвечать неограниченно возрастающая скорость движения шарнира $p_{j}$.

Второй пример - механизм Кемпе, чертящий распадающуюся на три прямых кривую $x(y-$ $1)(y-x-1)=0$. Все три прямые проходят через точку $(0,1)$. Этой точке отвечает положение $T=\left(0, \frac{7}{2}-\frac{\sqrt{3}}{2}\right)$ шарнира $D_{K}$, движущегося по оси ординат. Если шарнир $p_{j}$ движется по прямой $x=0$ с единичной скоростью из точки $(0,1)$, то шарнир $D_{k}$ движется из точки $T$ по оси ординат со скоростью $\frac{5}{2}-\frac{1}{\sqrt{3}} \approx 1,9226$. Если шарнир $p_{j}$ движется по прямой $y=1$ с единичной скоростью из точки $(0,1)$, то шарнир $D_{k}$ движется из точки $T$ по оси ординат со скоростью 1. А если шарнир $p_{j}$ движется по прямой $y=x+1$ с единичной скоростью из точки $(0,1)$, то шарнир $D_{k}$ движется из точки $T$ по оси ординат со скоростью $\frac{7 \sqrt{2}}{4}-\frac{\sqrt{6}}{6} \approx 2,0666$. Таким образом, передача движения от шарнира $D_{K}$ к чертящему шарниру $p_{j}$ неоднозначна в точке $D_{K}=T$, и для перехода с одной ветви рисуемой кривой на другую в точке $(0,1)$ должна скачком измениться скорость части механизма Кемпе.

Вторая претензия состоит в том, что используемые в доказательстве Кемпе антипараллелограммы могут вытягиваться и, распрямляясь, переходить в параллелограммы. Наоборот, шарнирный параллелограмм, распрямляясь, переходит в антипараллелограмм (см. рис. 11). Это может привести к появлению положений чертящего шарнира, не лежащих на алгебраической кривой, которую мы взялись чертить. Чтобы избежать таких паразитных положений, добавляют укрепляющие рычаги, препятствующие переходам параллелограмм - антипараллелограмм. Последнее 


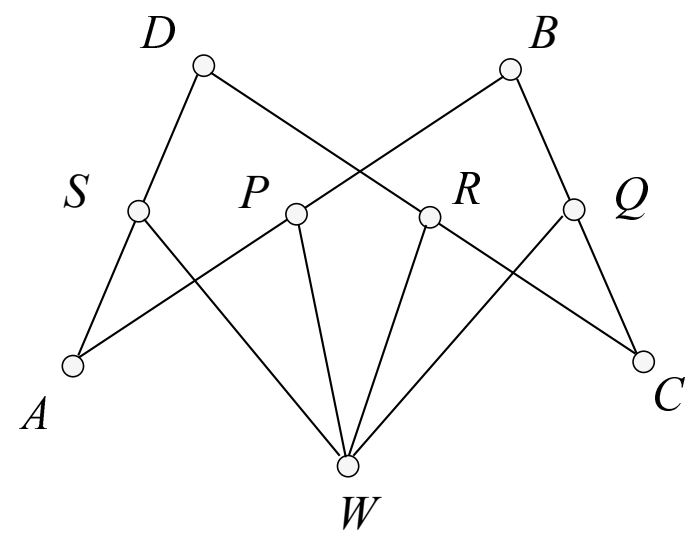

Рис. 12. Укрепленный антипараллелограмм. Шарниры $P, Q, R, S$ лежат посередине рычагов $A B$, $B C, C D$ и $D A$ антипараллелограмма.

особенно существенно при доказательстве теорем 2 и 3 о конфигурационном пространстве в целом. Например, параллелограмм можно укрепить, добавив к нему рычаг, соединяющий шарниры, расположенные в серединах его противоположных сторон (см. рис. 11).

Уточнением рассуждений Кемпе занимался ряд авторов, в частности, этому уделено много внимания в $[10,24]$. Чтобы избежать лишних положений рисующего алгебраическую кривую шарнира, были предложены укрепления элементарных механизмов-кирпичиков Кемпе. Так, в [10] доказано, что укрепленный рычагами $W P, W Q, W R, W S$ антипараллелограмм (см. рис. 12) нельзя собрать как параллелограмм. Это важно в рассуждениях Кемпе, ибо если складывать равные углы с помощью накопителя Кемпе, то антипараллелограмм, распрямляясь (без укрепляющих рычагов), мог бы перейти в параллелограмм, что влекло бы возникновение лишних точек в $K_{i}^{j} \cap U$. Как утверждают авторы работы [10], замена антипараллелограммов укрепленными антипараллелограммами исправляет рассуждения Кемпе и дает доказательство теоремы 5 в усиленном варианте, для проекции $K^{j}$ конфигурационного пространства КШС вместо проекции $K_{i}^{j}$ конфигурационного пространства шарнирного механизма.

Имеется еще третья претензия [24] к рассуждениям Кемпе, состоящая в том, что его построения выполнялись для острых углов. Она легко преодолима. Достаточно начало координат в доказательстве теоремы 1 взять так далеко от точки $p$, а ромб настолько вытянутым, чтобы углы $\varphi, \theta$ были так малы, что все их кратные в уравнении (1) лежали, скажем, в интервале $\left(-\frac{\pi}{4}, \frac{\pi}{4}\right)$. Максимальная кратность углов $\varphi, \theta$ в уравнении (1) равна степени многочлена, задающего кривую. Эта степень не меняется при невырожденной линейной подстановке переменных, отвечающей переходу к другой декартовой системе координат. Поэтому максимальная кратность углов равна порядку $N$ рисуемой алгебраической кривой. Если в теореме Кемпе ограничиться малыми движениями, то появление распрямленных антипараллелограммов возможно лишь для механизма накопителя при сложении углов, величины которых случайно совпали. Чтобы избежать этого, достаточно взять угол $\theta$ много меньшим угла $\varphi$, например, $\varphi>2 N \theta$. Чего можно добиться поворотом системы координат? Взяв теперь такую окрестность $U \subset \mathbb{R}^{2}$ точки $p$, чтобы для ромба с вершиной в произвольной точке $p^{\prime} \in U$ выполнялись условия

$$
N(\varphi+\theta)<\frac{\pi}{3}, \quad \varphi>N \theta
$$

мы можем быть уверены, что все механизмы-кирпичики построения Кемпе не вырождаются при движении шарнира $p_{j}$ внутри $U$.

С вышеприведенными уточнениями первоначальные рассуждения Кемпе представляют собой полное доказательство теоремы 7. Или, простыми словами, того факта, что произвольную ограниченную и связную часть алгебраической кривой можно без лишних точек вычертить шарнирным механизмом при его непрерывном движении. 


\section{СПИСОК ЛИТЕРАТУРЫ}

1. Артоболевский И. И. Теория механизмов. - М.: Наука, 1965.

2. Гилъберт Д., Кон-Фоссен С. Наглядная геометрия. - М.: Наука, 1981.

3. Горин E. А. Об асимптотических свойствах многочленов и алгебраических функций от нескольких переменных// Усп. мат. наук. - 1961. - 16, № 1 (97). - С. 91-118.

4. Ковалев М. Д. Геометрическая теория шарнирных устройств// Изв. РАН. Сер. мат. -1994. - 58, № 1. - C. $45-70$.

5. Ковалев М. Д. О восстановимости шарнирников по внутренним напряжениям// Изв. РАН. Сер. мат. - 1997. - 61, № 4. - С. 37-66.

6. Ковалев М. Д. Устойчивость шарнирников, шарнирных устройств и схем// Итоги науки и техн. Совр. мат. прилож. $-1999 .-68 .-$ С. $65-86$.

7. Ковалев М. Д. Вопросы геометрии шарнирных устройств и схем// Вестн. МГТУ. Сер. Машиностроение. - 2001. - № 4. - С. 33-51.

8. Левитский Н. И. Теория механизмов и машин. Терминология. - М.: Наука, 1984.

9. Ошемков А. А., Попеленский Ф. Ю., Тужилин А. А., Фоменко А. Т., Шафаревич А. И. Курс наглядной геометрии и топологии. - М.: ЛЕНАНД, 2015.

10. Abbott T. Generalizations of Kempe's Universality Theorem. - Cambridge, Massachusetts: Massachusetts Institute of Technology, 2008.

11. Asimov L., Roth B. The rigidity of Graphs. II// J. Math. Anal. Appl. - 1979. — 68, № 1. - P. 171-190.

12. Bottema O., Roth B. Theoretical Kinematics. - Amsterdam: North-Holland, 1979.

13. Crapo H. Structural Rigidity// Struct. Topol. — 1979. - № 1. - P. 26-45.

14. Demaine E., O'Rourke J. Geometric Folding Algorithms: Linkages, Origami, Polyhedra. — Cambridge Univ. Press, 2007.

15. Graver J., Servatius B., Servatius H. Combinatorial Rigidity. - Providence. Rhode Island: Am. Math. Soc., 1993.

16. Hunt K. H. Kinematic geometry of mechanisms. - New York: Clarendon Press, 1978.

17. Jordan D., Steiner M. Configuration spaces of mechanical linkages// Discr. Comput. Geom. - 1999. 22. - P. 297--315.

18. Kapovich M., Millson J. J. Universality theorems for configurations of planar linkages// Topology. — 2002. - 41, № 6. - P. 1051-1107.

19. Kempe A. B. On a general method of describing plane curves of the $n$th degree by Linkwork// Proc. London Math. Soc. - 1876. - 7, № 102. - P. 213-216.

20. King H. C. Planar linkages and algebraic sets/arXiv: 9807023 [math.AG] .

21. King H. C. Semiconfiguration spaces of planar linkages/arXiv: 9810130 [math.AG] .

22. King H. C. Configuration Spaces of Linkages in $R^{n} /$ arXiv: 9811138 [math. AG] .

23. Kovalev M. Results and problems of the geometry of hinged devices// Proc. 12th World Congress in Mechanism and Machine Science. - Besançon, France, 2007.

24. Power S. Elementary proofs of Kempe universality/arXiv: 1511.09002v2 [math.MG] .

Ковалев Михаил Дмитриевич

Московский государственный университет им. М. В. Ломоносова

E-mail: mdkovalev@mtu-net.ru 